\title{
A retrospective review of HIV PrEP offer and uptake in an Australian metropolitan sexual health centre
}

\author{
Andrew Phua ${ }^{1}$, Charlotte Bell ${ }^{2}$, Mahesh Ratnayake ${ }^{2}$
}

\begin{abstract}
Introduction: There is little data on offer and uptake of pre-exposure prophylaxis (PrEP) for HIV prevention on the ground.

Objective: The aim of this study was to assess the offer and uptake rates of PrEP in a metropolitan sexual health centre, among individuals at high risk for HIV infection. This can improve service delivery and uptake of HIV prevention.

Methods: A retrospective review was conducted among clients with a positive diagnosis of rectal gonorrhoea, rectal chlamydia, or infectious syphilis between 1 July to 31 December 2018, to ascertain what percentage were offered PrEP, and of those, what percentage took up PrEP.

Results: Of72 eligible males, 50 (69.4\%) were offered PrEP. Of these, 29 (58\%) took up PrEP, while 21 $(42 \%)$ did not. Those who declined often wanted time to consider, did not make an appointment, or declined due to side effects.

Conclusion: There is an acceptable PrEP offer rate which can be increased by training and enhanced documentation. More than half of eligible clients offered PrEP took it up, which is essential for HIV prevention. Patient education on PrEP and text reminders may increase PrEP uptake.
\end{abstract}

Key words:HIV prevention, HIV/AIDS, prevention, health services

Authors: ${ }^{1}$ Andrew Phua Sengkang General Hospital, Singapore, Singapore, ${ }^{2}$ Charlotte Bell, Adelaide Sexual Health Centre, Adelaide, Australia, ${ }^{2}$ Mahesh Ratnayake, Adelaide Sexual Health Centre, Adelaide, Australia Corresponding Author: Andrew Phua, drewphua@gmail.com

(c) (i)

Acknowledgement: The authors would like to thank Dr Alison Ward for input on the research direction, Mikko Li for assistance with data identification and extraction, and all staff at ASHC for their support and assistance with the research. This research did not receive any specific funding.

Conflict of interest: The authors declare no conflicts of interest, Funding: not funded, Originality: This is an original work which has been not published anywhere else 


\section{Full article}

\section{Introduction}

The combination of tenofovir and emtricitabine as human immunodeficiency virus (HIV) pre-exposure prophylaxis (PrEP) has been established as an effective prevention tool for HIV. $(1,2,3)$ PrEP was subsidised in April 2018 through the Australian Pharmaceutical Benefits Scheme (PBS), making it more accessible to Australians. (4) By the end of $2018,18,530$ people, $99 \%$ of them male, were receiving PBS-subsidised PrEP. (5) In addition to PrEP, non-occupational post-exposure prophylaxis (nPEP) has also seen increased use as treatment to prevent HIV spread after potential exposure to the virus. (6)

The Australasian Society for HIV, Viral Hepatitis and Sexual Health Medicine (ASHM) guidelines recommend offering PrEP to individuals at high risk for HIV infection, including those having unprotected anal intercourse or those with recent STI diagnoses. (8)

The Adelaide Sexual Health Centre (ASHC), as a metropolitan centre providing free and confidential specialist sexual health services in South Australia, sees many clients who fulfil eligibility criteria for PrEP. This audit examined what percentage of eligible clients at ASHC is offered PrEP. Of these, what percentage takes up PrEP? For those who don't, what are the reasons?

There is limited data on the actual offer of PrEP and decision-making around its uptake. It is hoped the findings can improve PrEP offer and uptake both locally and in other geographical contexts, thus reducing the transmission of HIV.

\section{Methods}

Ethics approval was sought and obtained to conduct a retrospective case notes review of clients eligible for PrEP in a single sexual health clinic, identified as men who had a diagnosis of rectal gonorrhoea, rectal chlamydia, or infectious syphilis within a six-month period between 1 July and 31 December 2018. This was deemed the most feasible criteria by which to identify eligible clients, guided by previous ASHM guidelines, and also due in part to the paucity of data for other risk factors. Records were anonymised and relevant information analysed, including diagnoses, demographic data, whether clients were offered PrEP, whether clients took up PrEP, and if not, the reasons for not taking up PrEP.

\section{Ethical considerations}

Ethics approval was gained from the Human Research Ethics Committee (HREC) of the Central Adelaide Local Health Network (CALHN) with project number HREC/18/CALHN/80.

\section{Results}

\section{Reasons for presentation}

The reason for performing STI screening for these clients may provide some context. The largest group was symptomatic clients seeking care $(37.4 \%)$, followed by asymptomatic clients requesting screening $(23.7 \%)$, clients on PrEP follow-up (16\%), clients called in as contacts of partners with positive STI diagnoses (15.3\%), clients requesting nPEP (5.3\%), and clients being referred, seen in HIV clinic, and seen post-sexual assault (1\% each). This is seen in Figure 1 below.

Figure 1: Reasons for clients' presentation and screening

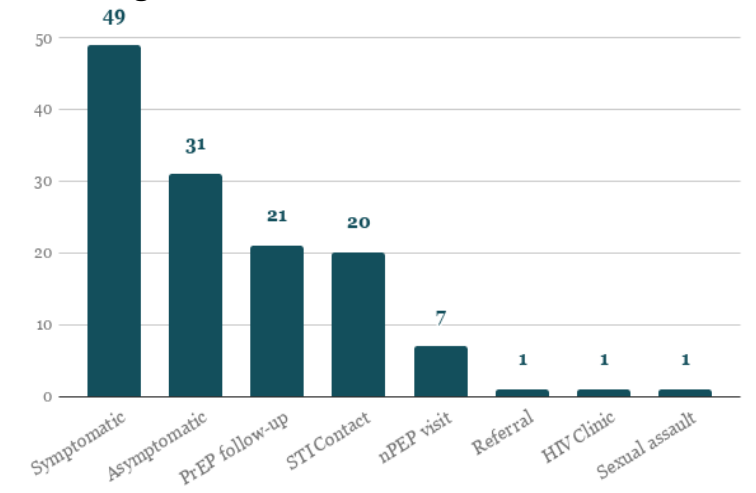

\section{Diagnoses}

There were 142 diagnoses of rectal gonorrhoea, rectal chlamydia, or infectious syphilis between 1 July and 31 December 2018 from 131 male clients (see Figure 2). 
Figure 2: Breakdown of 142 diagnoses identifying eligible clients

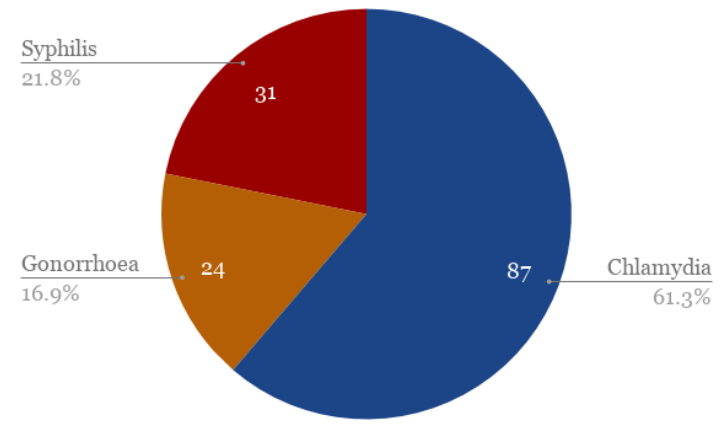

From Figure 2, of 142 STI diagnoses during the study period, 87 were from rectal chlamydia (62.2\%), 24 were from rectal gonorrhoea $(16.9 \%)$, and 31 were from infectious syphilis (20.9\%).

\section{Demographics}

The demographic data of the 131 clients identified is presented below.

Table 1: Selected demographic data of eligible clients

\begin{tabular}{|c|c|}
\hline Characteristic & Value \\
\hline Age & $34.8 \pm 11.8$ \\
\hline Born in Australia & 95 (72.5\%) \\
\hline \multicolumn{2}{|c|}{ Choice of Sexual Partners: } \\
\hline Same Sex & $85(64.9 \%)$ \\
\hline Opposite Sex & $3(2.3 \%)$ \\
\hline Both Sexes & $40(30.5 \%)$ \\
\hline Not Answered & $3(2.3 \%)$ \\
\hline \multicolumn{2}{|c|}{ Number of STIs diagnosed in the past year: } \\
\hline 1 & $62(47.3 \%)$ \\
\hline 2 & $36(27.5 \%)$ \\
\hline 3 & $20(15.3 \%)$ \\
\hline 4 & $7(5.3 \%)$ \\
\hline 5 & $3(2.3 \%)$ \\
\hline 6 & $3(2.3 \%)$ \\
\hline
\end{tabular}

The mean age of eligible clients was 34.8 years, with the majority $(72.5 \%)$ born in Australia. Almost two-thirds $(64.9 \%)$ of eligible clients answered their sexual partners are of the same sex, while $30.5 \%$ answered their sexual partners are of both sexes, with an equal minority stating their sexual partners are of the opposite sex or declining to answer.
In addition, almost half (47.3\%) of eligible clients had a single STI diagnosis in the past year, followed by $27.5 \%$ having two STI diagnoses, with a smaller percentage having three or more STI diagnoses. This shows a significant pool of clients having multiple STIs through a given year, which is an opportunity for education about safe sex practices and HIV prophylaxis.

\section{PrEP uptake}

Figure 3: Flowchart of identifying and offering PrEP to eligible clients

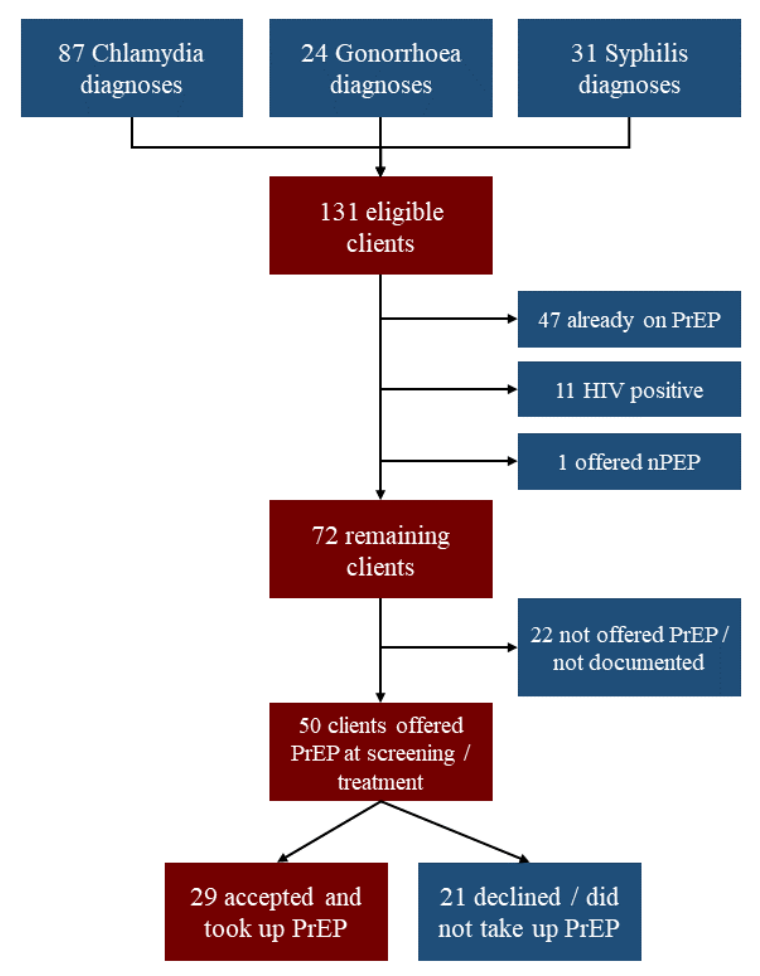

Of the 131 eligible clients identified, 47 were already on PrEP, 11 were HIV positive therefore not eligible for PrEP, and 1 was offered nPEP instead. Of the remaining 72 eligible clients, 50 (69.4\%) were offered PrEP either at screening visit or at results/treatment visit. Of these, 29 (58\%) accepted and took up PrEP, while 21 (42\%) either declined PrEP or did not accept the offer.

Of those who declined, some said they would consider (8; 38.1\%), others agreed but did not make an appointment $(4 ; 19 \%)$, some declined due to side effects $(3 ; 14.3 \%)$ or were not interested $(3 ; 14.3 \%), 2$ had no reason 
documented (9.5\%), and 1 cited cost as a reason $(4.8 \%)$.

\section{Discussion}

This audit found an acceptable PrEP offer rate to eligible clients. However, there is still a significant portion of eligible clients not being offered PrEP. This could be due to a few reasons. One is that some clients present as sexual contacts of clients who have had a positive STI diagnosis. These contacts receive STI screening and also empirical treatment for the STI at the initial visit. Later on, they may test positive for an STI, but having been already empirically treatment at their prior visit, they are not recalled and hence do not have a visit opportunity where PrEP can be discussed. In these cases, standardised sexual history-taking should reveal clients' eligibility, whereupon PrEP can be discussed and offered.

Another reason could be staff being unfamiliar with the PrEP guidelines, due to regular rotations of medical doctors at the ASHC. This can be addressed with regular standardised training to all staff on the eligibility criteria for offering PrEP to clients, especially given the periodic updates published by ASHM.

Another possibility is that PrEP was discussed or offered during a client's visit, but was not documented. For clients that eventually take up PrEP, this may not be an issue, but for clients who decline PrEP, the lack of documentation makes it appear there has been a lost opportunity to offer PrEP to an eligible client. Hence, enhanced documentation of eligibility can improve PrEP offer and uptake rates.

Aside from increasing offer rates, it is worthwhile to discuss how to increase PrEP uptake after it has been offered. More than half of eligible clients who were offered PrEP took it up, which is essential for HIV prevention. Nevertheless, $42 \%$ of those offered PrEP eventually declined or did not take up PrEP. A large percentage of clients offered PrEP said they would consider it, but eventually did not take it up. It may be helpful to set a system reminder to check back in with the same client about their decision regarding PrEP. Text reminders may help to increase PrEP uptake, by reminding clients who agreed to consider or take up PrEP but did not follow through.

Other clients mentioned negative side effects as a reason not to take up PrEP. In some cases, these are perceived side effects. Hence, more information around PrEP and how it can be taken, in addition to literature around sideeffects, can potentially mitigate clients' negative impressions on PrEP. For example, different PrEP dosing regimes can maintain efficacy while reducing side effects, and informing clients of such regimes may increase uptake.

This review of PrEP offer and uptake in a metropolitan sexual health service centre in Australia shows an acceptable but improvable rate of PrEP offer to eligible clients, and moderate PrEP uptake rate when offered. It also reveals that PrEP offer rates can be improved through staff training and improved documentation. Other sexual health service providers can gain from our experience and consider how best to improve PrEP offer and uptake rates in their local context.

\section{References}

1. Hammoud MA, Vaccher S, Jin F, et al. HIV Preexposure Prophylaxis (PrEP) Uptake Among Gay and Bisexual Men in Australia and Factors Associated With the Nonuse of PrEP Among Eligible Men : Results From a Prospective Cohort Study. JAIDS Journal of Acquired Immune Deficiency Syndromes. July 2019;81(3):e73-e84.

2. Anderson P, Glidden D, Liu A, et al. Emtricitabinetenofovir concentrations and pre-exposure prophylaxis efficacy in men who have sex with men. Science translational medicine. September 2012;4(151):151ra125.

3. Fonner A,V, Dalglish L,S, Kennedy E,C, et al. Effectiveness and safety of oral HIV preexposure prophylaxis for all populations. AIDS. 2016;30(12):1973-1983.

4. National Prescriber Schedule. PrEP on the PBS: An opportunity in HIV prevention. NPS Medicinewise [News]. April 16, 2018. Available at: https://www.nps.org.au/news/pr-ep-on-the-pbs-anopportunity-in-hiv-prevention. Accessed August 2, 2019.

5. Kirby Institute. Monitoring HIV pre-exposure prophylaxis in Australia. Sydney: Kirby Institute; 2019. 
6. Minas B, Laing S, Jordan H, Mak DB. Improved awareness and appropriate use of non-occupational post-exposure prophylaxis (nPEP) for HIV prevention following a multi-modal communication strategy. BMC Public Health. October 2012;12:906.

7. ASHM. The Australasian Society of HIV, Viral Hepatitis and Sexual Health Medicine (ASHM) PrEP Guidelines Update. Prevent HIV by Prescribing PrEP. 2019 ed. Sydney; 2019.

8. Wright E, Grulich A, Roy K, et al. Australasian Society for HIV, Viral Hepatitis and Sexual Health Medicine HIV pre-exposure prophylaxis: clinical guidelines. Update April 2018. Journal of Virus Eradication. April 2018;4(2):143-159. 